
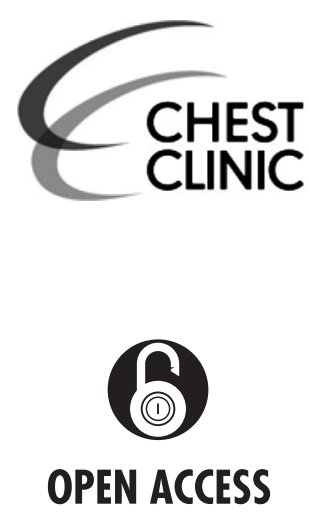

For numbered affiliations see end of article.

\section{Correspondence to} Dr R Andres Floto, Cambridge Institute for Medical Research, University of Cambridge, Cambridge Biomedical Campus, Hills Road, Cambridge CB2 OXY, UK; arf27@cam.ac.uk

Received 26 October 2015 Accepted 5 November 2015

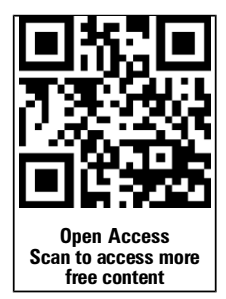

\section{SLinked}

- http://dx.doi.org/10.1136/ thoraxjnl-2015-207360

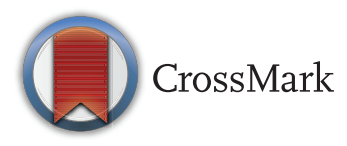

To cite: Floto RA, Olivier KN, Saiman L, et al. Thorax 2016;71:88-90.

\title{
US Cystic Fibrosis Foundation and European Cystic Fibrosis Society consensus recommendations for the management of non-tuberculous mycobacteria in individuals with cystic fibrosis: executive summary
}

\author{
R Andres Floto, ${ }^{1,2}$ Kenneth N Olivier, ${ }^{3}$ Lisa Saiman, ${ }^{4}$ Charles L Daley, ${ }^{5}$ \\ Jean-Louis Herrmann, ${ }^{6,7}$ Jerry A Nick, ${ }^{8}$ Peadar G Noone, ${ }^{9}$ Diana Bilton, ${ }^{10}$ \\ Paul Corris, ${ }^{11}$ Ronald L Gibson, ${ }^{12}$ Sarah E Hempstead, ${ }^{13}$ Karsten Koetz, ${ }^{14}$ \\ Kathryn A Sabadosa, ${ }^{13}$ Isabelle Sermet-Gaudelus, ${ }^{15}$ Alan R Smyth, ${ }^{16}$ \\ Jakko van Ingen, ${ }^{17}$ Richard J Wallace, ${ }^{18}$ Kevin L Winthrop, ${ }^{19}$ Bruce C Marshall, ${ }^{20}$ \\ Charles S Haworth ${ }^{2}$
}

\section{ABSTRACT}

Non-tuberculous mycobacteria (NTM) are ubiquitous environmental organisms that can cause chronic pulmonary infection, particularly in individuals with preexisting inflammatory lung disease, such as cystic fibrosis (CF). Pulmonary disease (PD) caused by NTM has emerged as a major threat to the health of individuals with CF, but remains difficult to diagnose and problematic to treat. In response to this challenge, the US Cystic Fibrosis Foundation (CFF) and the European Cystic Fibrosis Society (ECFS) convened a panel of 19 experts to develop consensus recommendations for the screening, investigation, diagnosis and management of NTM-PD in individuals with CF. PICO (population, intervention, comparison, outcome) methodology and systematic literature reviews were employed to inform draft recommendations, which were then modified to achieve consensus and subsequently circulated for public consultation within the USA and European CF communities. We have thus generated a series of pragmatic, evidence-based recommendations as an initial step in optimising management for this challenging condition.

\section{BACKGROUND}

Non-tuberculous mycobacteria (NTM) are increasingly being isolated from the sputum of adults and children with cystic fibrosis (CF) both in North America and Europe. Estimates of the prevalence of NTM in the CF population have ranged from $1.3 \%$ in the earliest study reported in $1984^{1}$ to $32.7 \%$ in a review of patients with CF over age 40 in Colorado. ${ }^{2}$

The NTM species most commonly identified in individuals with CF from North America and Europe are the slow growing Mycobacterium avium complex (MAC, including M. avium, M. intracellulare and M. chimaera), which can be found in up to $72 \%$ of NTM-positive sputum cultures, and the rapid growing M. abscessus complex (comprising the subspecies M. abscessus subsp abscessus (M. a. abscessus), M. a. bolletii and M. a. massiliense (the latter currently classified as part of M. a. bolletii)), which in many centres has now become the most common NTM isolated from individuals with $\mathrm{CF}$.

There has been a rise in the prevalence of NTM-positive cultures in respiratory samples from individuals with $\mathrm{CF}$ over the last three decades, which probably reflects a true increase in the frequency of NTM infection. A number of CF studies (eg, Renna $e t a l^{3}$ ) show year-on-year increases in NTM-positive cultures with no change in surveillance intensity or culture methodology.

Possible reasons for increased NTM-positive cultures in individuals with $\mathrm{CF}$ include: increases in environmental exposure to NTM through more permissive temperature settings of home water heaters and more contact with shower aerosols, increased antibiotic usage creating more NTM permissive lung niches, greater chronic use of medications that might impair host immunity to $\mathrm{NTM}^{3}$ and/or spread of NTM through person-to-person transmission. ${ }^{4}$

NTM can cause progressive inflammatory lung damage, a condition termed 'NTM pulmonary disease' (NTM-PD), which is defined by the presence of specific microbiological, clinical and radiological features. ${ }^{5}$ However, it has become clear that NTM can also transiently, intermittently or permanently reside within the lungs of $\mathrm{CF}$ individuals without causing NTM-PD, thus representing asymptomatic infection and creating considerable difficulties in deciding how best to screen for and diagnose NTM.

Further challenges exist in knowing how best to identify NTM in respiratory samples, when and how to initiate treatment for NTM-PD and how NTM may impact individuals under consideration for lung transplantation. As a consequence, the Cystic Fibrosis Foundation (CFF) and European Cystic Fibrosis Society (ECFS) sought to generate a consensus recommendations document to support and standardise the management of NTM infection in children and adults with CF, permitting 
prospective evaluation of current best practice and forming a foundation for future research programmes.

\section{METHODS}

The CFF and the ECFS invited experts to participate in the statement development process. The 19 member committee consisted of professionals with expertise in CF and NTM and included adult and paediatric CF physicians, lung transplant physicians, microbiologists, infectious disease specialists and a parent of an individual with CF. The committee convened in May 2012 and divided into five subgroups, each responsible for a specific topic: epidemiology and risk factors, screening,

\begin{tabular}{|c|c|}
\hline \multirow{4}{*}{ 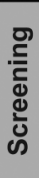 } & $\begin{array}{l}\text { The potential for cross-infection of NTM (particularly } M \text {. abscessus complex) between individuals with CF should be minimised by following nati } \\
\text { fection control guidelines. }\end{array}$ \\
\hline & Cultures for NTM be performed annually in spontaneously expectorating individuals with a stable clinical course. \\
\hline & $\begin{array}{l}\text { 3. In the absence of clinical features suggestive of NTM pulmonary disease, individuals who are not capable of spontaneously producing sputum do not } \\
\text { require screening cultures for NTM. }\end{array}$ \\
\hline & $\begin{array}{l}\text { 4. Culture and smears for acid fast bacilli from sputum should be used for NTM screening. } \\
\text { 5. Oro-pharyngeal swabs should not be used for NTM screening. }\end{array}$ \\
\hline \multirow{14}{*}{$\begin{array}{l}\text { 정 } \\
\text { 응 } \\
\frac{0}{0} \\
\text { 인 } \\
\text { 을 }\end{array}$} & $\begin{array}{l}\text { 6. Cultures and smears for acid fast bacilli (AFB) from sputum, induced sputum, bronchial washings or broncho-alveolar lavage samples can be used to } \\
\text { evaluate individuals with CF suspected to have NTM pulmonary disease. }\end{array}$ \\
\hline & 7. Transbronchial biopsies should not be routinely used to detect NTM in individuals with CF suspected to have NTM pulmonary disease. \\
\hline & 8. Oro-pharyngeal swabs should not be used to perform diagnostic smears and cultures in individuals with CF suspected to have NTM pulmonary disease. \\
\hline & 9. Respiratory tract samples should be cultured using both solid and liquid media. \\
\hline & 10. The incubation duration for NTM cultures should be for a minimum of 6 weeks. \\
\hline & $\begin{array}{l}\text { 11. An NTM culture should be processed within } 24 \text { hours of collection to optimize the detection of NTM in respiratory samples. If a delay in processing is } \\
\text { anticipated, refrigeration of samples is advised. }\end{array}$ \\
\hline & 12. Respiratory tract samples should be decontaminated using the standard N-Acetyl L-cysteine NALC $(0.5 \%)-\mathrm{NaOH}(2 \%)$ method. \\
\hline & $\begin{array}{l}\text { 13. If a sample remains contaminated with gram-negative bacteria after standard NALC-NaOH decontamination, it should be further treated with either } 5 \% \\
\text { oxalic acid or } 1 \% \text { chlorhexidine. }\end{array}$ \\
\hline & 14. Non-culture based methods should not be used for detecting NTM in respiratory tract samples. \\
\hline & 15. All NTM isolates from individuals with CF should undergo molecular identification. \\
\hline & $\begin{array}{l}\text { 16. All NTM isolates from individuals with CF should be identified to the species level, except for } M \text {. intracellulare, } M \text {. avium and } M \text {. chimaera, where } \\
\text { identification can be limited to } M \text {. avium complex (MAC), and } M \text {. abscessus complex, which should be sub-speciated. }\end{array}$ \\
\hline & $\begin{array}{l}\text { 17. For } M \text {. avium complex, clarithromycin susceptibility testing should be performed on an isolate recovered prior to initiation of treatment. Clarithromycin } \\
\text { susceptibility testing should also be performed on subsequent isolates if the patient a) fails to culture convert after six months of NTM treatment; b) } \\
\text { recultures } M \text {. avium complex after initial culture conversion while on NTM treatment; or c) recultures } M \text {. avium complex after completion of NTM treatment. }\end{array}$ \\
\hline & $\begin{array}{l}\text { 18. For } M \text {. abscessus complex, susceptibility testing should include at least clarithromycin, cefoxitin and amikacin (c } \\
\text { imipenem, minocycline, moxifloxacin and linezolid). }\end{array}$ \\
\hline & \\
\hline \multirow{4}{*}{ 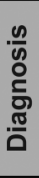 } & ividuals with CF \\
\hline & $\begin{array}{l}\text { 21. Other CF pathogens and co-morbidities should be considered as potential contributors to a patient's symptoms and radiological features when } \\
\text { determining the clinical significance of NTM positive cultures. }\end{array}$ \\
\hline & 22. NTM treatment should be considered for individuals with CF who have ATS/IDSA defined NTM pulmonary disease. \\
\hline & $\begin{array}{l}\text { 23. Individuals receiving azithromycin as part of their CF medical regimen who have a positive NTM culture should not continue azithromycin treatment } \\
\text { while evaluation for NTM disease is underway as azithromycin monotherapy may lead to resistance. A macrolide agent may be included in a multi-drug } \\
\text { treatment regimen if criteria are met for NTM disease. }\end{array}$ \\
\hline \multirow{20}{*}{ 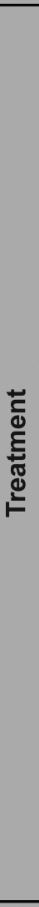 } & $\begin{array}{l}\text { 24. Treatment of } M \text {. abscessus complex pulmonary disease should involve an intensive phase followed by a continuation phase. } \\
\text { 25. The intensive phase should include a daily oral macrolide (preferably azithromycin) in conjunction with } 3-12 \text { weeks of intravenous amikacin and one or } \\
\text { more of the following: intravenous tigecycline, imipenem or cefoxitin, guided but not dictated by drug susceptibility testing. The duration of intensive phase } \\
\text { therapy should be determined by the severity of infection, the response to treatment and the tolerability of the regimen. }\end{array}$ \\
\hline & $\begin{array}{l}\text { 26. The continuation phase should include a daily oral macrolide (preferably azithromycin) and inhaled amikacin, in conjunction with 2-3 of the following } \\
\text { additional oral antibiotics: minocycline, clofazimine, moxifloxacin and linezolid, guided but not dictated by drug susceptibility testing. }\end{array}$ \\
\hline & $\begin{array}{l}\text { 27. Individuals with } M \text {. abscessus complex pulmonary disease should be managed in collaboration with experts in the treatment of NTM and CF as drug } \\
\text { intolerance and drug-related toxicity occur frequently and changes in antibiotic therapy are often required. }\end{array}$ \\
\hline & 28. Monotherapy with a macrolide or other antimicrobial should never be used in the treatment of $M$. abscessus complex pulmonary disease. \\
\hline & 29. The same antibiotic regimen should be used for treatment of all species within the M. avium complex. \\
\hline & $\begin{array}{l}\text { 30. Clarithromycin-sensitive } M \text {. avium complex pulmonary disease should be treated with a daily oral antibiotic regimen containing a macrolide (preferably } \\
\text { azithromycin), rifampin and ethambutol. }\end{array}$ \\
\hline & ree-times-per week) oral antibiotic therapy should not be used to treat $M$. avium complex pulmonary disease. \\
\hline & 32. Monotherapy with a macrolide or other antimicrobial agent should never be used in the treatment of $M$. avium complex pulmonary disease. \\
\hline & $\begin{array}{l}\text { 33. An initial course of intravenous amikacin should be considered for the treatment of } M \text {. avium complex pulmonary disease in the presence of one or } \\
\text { more of the following: i)AFB smear positive respiratory tract samples; ii) Radiological evidence of lung cavitation or severe infection; iii) Systemic signs of } \\
\text { illness. }\end{array}$ \\
\hline & 34. Clarithromycin-resistant M. avium complex pulmonary disease should be managed in collaboration with experts in the treatment of NTM and CF. \\
\hline & $\begin{array}{l}\text { 35. Individuals with CF receiving NTM treatment should have expectorated or induced sputum samples sent for NTM culture every } 4-8 \text { weeks throughout } \\
\text { the entire course of treatment to assess the microbiological response. }\end{array}$ \\
\hline & $\begin{array}{l}\text { 36. A schedule for detecting drug toxicity (including hearing loss, visual loss, renal impairment and liver function test abnormalities) should be set in place } \\
\text { at the time of NTM treatment initiation and implemented throughout treatment based on the specific drugs prescribed. }\end{array}$ \\
\hline & $\begin{array}{l}\text { 37. An HRCT scan of the lungs should be performed shortly before starting NTM treatment and at the end of NTM treatment to assess the radiological } \\
\text { response. }\end{array}$ \\
\hline & $\begin{array}{l}\text { M antibiotic therapy should be prescribed for } 12 \text { months beyond culture conversion (defined as three consecutive negative cultures, with the time of } \\
\text { rsion being the date of the first of the three negative cultures) as long as no positive cultures are obtained during this } 12 \text { months. }\end{array}$ \\
\hline & 39. Individuals who fail to culture convert despite optimal NTM therapy may benefit from long term suppressive antibiotic treatment. \\
\hline & $\begin{array}{l}\text { 40. When amikacin is given intravenously or when streptomycin is given intravenously or intramuscularly, serum levels should be monitored and dosing } \\
\text { adjusted to minimize ototoxicity and nephrotoxicity. }\end{array}$ \\
\hline & $\begin{array}{l}\text { 41. Serum levels of other anti-mycobacterial drugs should not be routinely obtained. However, absorption of oral medications is often reduced in CF. } \\
\text { Therefore use of therapeutic drug monitoring should be considered for individuals failing to improve despite taking recommended drug regimens or for } \\
\text { those on concomitant medications with significant interactions with NTM drugs. }\end{array}$ \\
\hline & 42. Interferon gamma should not be used as adjuvant therapy for NTM pulmonary disease in individuals with CF. \\
\hline & 43. Vitamin D should be supplemented according to national CF care guidelines. \\
\hline & 44. Lung resection should only be considered in extraordinary circumstances and in consultation with experts in the treatment of NTM and CF. \\
\hline \multirow{6}{*}{ 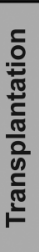 } & 45. All individuals with CF being considered for lung transplantation should be evaluated for NTM pulmonary disease. \\
\hline & $\begin{array}{l}\text { 46. The presence of current or previous respiratory tract samples positive for NTM should not preclude individuals being considered for lung } \\
\text { transplantation. }\end{array}$ \\
\hline & $\begin{array}{l}\text { 47. Individuals with CF who have NTM pulmonary disease and are being evaluated for transplantation should commence treatment prior to transplant } \\
\text { listing. }\end{array}$ \\
\hline & 48. Individuals with CF receiving NTM treatment with sequential negative cultures may be eligible for transplant listing. \\
\hline & $\begin{array}{l}\text { 49. Individuals with CF who have completed treatment for NTM pulmonary disease with apparent eradication of the organism may be eligible for transplant } \\
\text { listing. }\end{array}$ \\
\hline & 50. The presence of persistent $M$. abscessus complex or $M$. avium complex infection despite optimal therapy is not an absolute contraindication to lung \\
\hline
\end{tabular}

Figure 1 Cystic Fibrosis Foundation and European Cystic Fibrosis Society recommendations on non-tuberculous mycobacteria (NTM) management in cystic fibrosis (CF). 
microbiology, treatment and transplantation. Each subgroup developed topic-specific questions using the PICO format (population, intervention, comparison, outcome). Questions were reviewed and approved by the entire committee before systematic literature searches were conducted.

The members of each subgroup used the PICO questions to guide literature searches in PubMed. Searches were limited to English language and the period 1984-2013. Subgroup members also searched for topic relevant guidelines through searches of the ATS website, the IDSA website, the Clinical Laboratory Standards Institute website and the UK CF Trust website.

After reviewing relevant literature and existing guidelines, subgroup members drafted recommendation statements. In October 2012, a second meeting was convened, and subgroups finalised draft recommendation statements. The committee also voted to set $80 \%$ agreement of all 19 members as the threshold for acceptance of a recommendation statement.

Each subgroup submitted final draft questions for entry into an electronic survey tool (Survey Monkey) for the purposes of anonymous voting and comment by all members. A project coordinator administered the survey, and committee members were asked to rate each statement on a scale of 0 (completely disagree) to 9 (completely agree), with $80 \%$ or between 7 and 9 being considered 'good' agreement. Space for entering free text was also provided after each statement to allow members to cite literature in support of their opinions or suggested revisions. All committee members were required to vote on each statement regardless of their role or expertise. Multiple rounds of voting and revisions to the statements were conducted, and for each round committee members were requested to complete their voting within 3 weeks. The committee chairs reviewed the results from each round and updated the statements based on comments entered by respondents for subsequent rounds.

A draft of the recommendations was presented at the 2013 North American Cystic Fibrosis Conference and the ECFS Meeting. In addition, the committee solicited feedback from the CF communities in the USA and Europe, which included physicians, nurses, physical and respiratory therapists, parents and individuals with CF. Comments collected from this process were considered by the committee in the development of the final recommendation statements.

\section{RESULTS}

Three rounds of voting were conducted to achieve $80 \%$ consensus for each statement. Fifty-three statements were included in the first round of voting and 50 statements in the second and third rounds. Final statements are shown in figure 1.

\section{DISCUSSION}

The management of individuals with CF infected with NTM is extremely challenging. The limited amounts of published research and clinical trial data provide inadequate evidence to base management decisions on how best to screen, diagnose, detect and treat NTM-PD. As a response to this urgent clinical need, the CF Foundation and ECFS formed a committee of clinicians, scientists and infectious disease experts to develop recommendations to guide and assist clinicians in the management of NTM-PD in individuals with CF. The committee believe these recommendations should serve as a benchmark for current medical care while providing a framework to inform the development of clinical, translation and basic research studies to generate robust evidence to base future iterations of these management guidelines leading to better outcomes for individuals with CF infected with NTM.

\section{Author affiliations}

${ }^{1}$ Cambridge Institute for Medical Research, University of Cambridge, Cambridge, UK ${ }^{2}$ Cambridge Centre for Lung Infection, Papworth Hospital, Cambridge, UK

${ }^{3}$ Cardiovascular and Pulmonary Branch, National Heart, Lung, and Blood Institute, $\mathrm{NIH}$, Bethesda, Maryland, USA

${ }^{4}$ Department of Pediatrics, Columbia University Medical Center, Pediatric Infectious Diseases, New York, New York, USA

${ }^{5}$ Division of Mycobacterial and Respiratory Infections, National Jewish Health, Denver, Colorado, USA

${ }^{6}$ INSERM U1173, UFR Simone Veil, Versailles-Saint-Quentin University, Saint-Quentin en Yvelines, France

${ }^{7}$ AP-HP, Service de Microbiologie, Hôpital Raymond Poincaré, Garches, France

${ }^{8}$ Department of Medicine, National Jewish Health, Denver, Colorado, USA

${ }^{9}$ Division of Pulmonary and Critical Care Medicine, The University of North Carolina at Chapel Hill, Chapel Hill, North Carolina, USA

${ }^{10}$ Department of Respiratory Medicine, Royal Brompton Hospital, London, UK

${ }^{11}$ Department of Respiratory Medicine, Freeman Hospital High Heaton, Newcastle, UK

${ }^{12}$ Department of Pediatrics, University of Washington School of Medicine, Seattle, Washington, USA

${ }^{13}$ The Dartmouth Institute for Health Policy and Clinical Practice, Geisel School of Medicine at Dartmouth, Lebanon, New Hampshire, USA

${ }^{14}$ Department of Pediatrics, Sahlgrenska University Hospital, Gothenburg, Sweden

${ }^{15}$ Service de Pneumo-Pédiatrie, Université René Descartes, Hôpital Necker-Enfants Malades, Paris, France

${ }^{16}$ Division of Child Health, Obstetrics \& Gynaecology, University of Nottingham, Nottingham, UK

${ }^{17}$ Department of Medical Microbiology, Radboud University Medical Center, Nijmegen, The Netherlands

${ }^{18}$ Department. of Microbiology, University of Texas Health Science Center, Tyler, Texas, USA

${ }^{19}$ Divisions of Infectious Diseases, Public Health and Preventive Medicine, Oregon Health and Science University, Portland, Oregon, USA

${ }^{20}$ Cystic Fibrosis Foundation, Bethesda, Maryland, USA

Contributors All authors contributed to the expert committee on guidelines development (cochairs: RAF, CSH; steering committee: RAF, CSH, BCM, KNO, LS, KAS, SEH); committee subgroups: epidemiology and risk factors (KNO (lead), IS-G, KLW); screening (JAN (lead), RLG, KK); microbiology (J-LH (lead), RJW, Jvl, RAF); treatment (CLD (lead), DB, LS, ARS, CSH) and transplantation (PGN (lead), PC).

Funding Cystic Fibrosis Foundation; European Cystic Fibrosis Society, The Wellcome Trust \& Cambridge NIHR BRC (RAF); Intramural programme of the National Heart, Lung, and Blood Institute, NIH (KNO); Vaincre La Mucoviscidose (VLMIC1014 and RF20120600689) and the Région Ile-de-France Domaine d'Intérêt Majeur Maladies Infectieuses et Emergentes (J-LH); CF Foundation Clinical Research Award (NICK13A0) (JAN) and Imperial College London NIHR Respiratory BRU (DB).

Competing interests None declared.

Patient consent Obtained.

Provenance and peer review Not commissioned; internally peer reviewed.

Open Access This is an Open Access article distributed in accordance with the terms of the Creative Commons Attribution (CC BY 4.0) license, which permits others to distribute, remix, adapt and build upon this work, for commercial use, provided the original work is properly cited. See: http://creativecommons.org/ licenses/by/4.0/

\section{REFERENCES}

1 Smith MJ, Efthimiou J, Hodson ME, et al. Mycobacterial isolations in young adults with cystic fibrosis. Thorax 1984:39:369-75.

2 Rodman DM, Polis JM, Heltshe SL, et al. Late diagnosis defines a unique population of long-term survivors of cystic fibrosis. Am J Respir Crit Care Med 2005;171:621-6.

3 Renna M, Schaffner C, Brown K, et al. Azithromycin blocks autophagy and may predispose cystic fibrosis patients to mycobacterial infection. J Clin Invest 2011:121:3554-63.

4 Bryant JM, Grogono DM, Greaves D, et al. Whole-genome sequencing to identify transmission of mycobacterium abscessus between patients with cystic fibrosis: A retrospective cohort study. Lancet 2013;381:1551-60.

5 Griffith DE, Aksamit T, Brown-Elliott BA, et al. An official ATS/IDSA statement: diagnosis, treatment, and prevention of nontuberculous mycobacterial diseases. Am J Respir Crit Care Med 2007;175:367-416. 